\title{
Enhancement of Fundamental Mode Third Harmonic Generation Efficiency in Microfibres
}

\author{
Xiujuan Jiang ${ }^{1,2}$, Timothy Lee ${ }^{2}$, and Gilberto Brambilla ${ }^{2}$ \\ ${ }^{1}$ School of Electro-mechanical Engineering, Guangdong University of Technology, Guangzhou, 510006, China \\ ${ }^{2}$ Optoelectronics Research Centre, University of Southampton, Southampton, SO17 1BJ, United Kingdom \\ jiangxj@gdut.edu.cn
}

\begin{abstract}
We present a scheme to generate the third harmonic in the fundamental mode using microfibres, by introducing a counter-propagating pump field to partly compensate the phase mismatch and thus enhance the harmonic conversion efficiency.
\end{abstract}

OCIS codes: $190.2620,190.4370$.

\section{Introduction}

Third harmonic generation (THG) remains an important area of research, with numerous applications. For example, with high-power laser drivers, such as the Omega Facility in Rochester University, USA and Shenguang II Facility at the Shanghai Institute of Optics and Fine Mechanics, China, THG is used to convert an infra-red pump into ultra-violet wavelengths, as the latter have a higher energy coupling efficiency in laser-plasma interactions. The systems for THG utilizing crystals are usually complicated because many optical components are required. In recent years however, harmonic generation in microfibres has been reported [1], thus opening the possibility for an all-fibre system for THG as a more robust alternative to the existing nonlinear-crystal technologies.

As with other nonlinear harmonic conversion processes, phase matching should be satisfied to attain efficient THG in microfibres. However, because of material and waveguide dispersion, only intermodal phase matching can be achieved directly in glass microfibres (i.e. between the fundamental pump mode and a higher order harmonic mode [2,3]) and no efforts on generating the fundamental third harmonic mode in microfibres have been reported to date. Nonetheless, a fundamental mode output is often desired when developing a laser, because its nearly Gaussian field distributions would be easily compatible with other laser sources and equipment, and better for irradiation smoothing. In this paper, we therefore present a scheme in which the phase mismatch is partly compensated by introducing a counterpropagating pump field into the microfibre, thereby allowing the harmonic to be generated in the fundamental mode.

\section{Basic principles}

In the process of THG, the total electric field can be represented as a sum of the mode fields at each frequency $\omega_{j}$

$$
\mathbf{E}(x, y, z, t)=\sum \mathbf{E}_{j}(x, y, z, t)=\sum A_{j}(z) \mathbf{F}_{j}(x, y) \exp \left[i\left(\beta_{j} z-\omega_{j} t\right)\right]+c . c .,
$$

where $j=1$ for the pump and $j=3$ for the third harmonic; $\mathbf{F}_{j}(x, y)$ are the electric mode field distributions and $A_{j}(z)$ are the amplitudes of the modes normalised to their powers, that is, $\left|A_{j}(z)\right|^{2}=P_{j}(z) . \beta_{j}$ are the propagation constants of the modes in the fibre defined as $\beta_{j}=\left(\omega_{j} / c\right) n_{j}^{e f f}\left(\omega_{j}\right)$, and $n_{j}^{\text {eff }}$ are the effective indices of the modes.

In step-index glass-air circular fibres, the following coupled-mode equations can be used to describe the THG process [1],

$$
\begin{aligned}
& \frac{\partial A_{1}}{\partial z}=i n^{(2)} k_{1}\left[\left(J_{1}\left|A_{1}\right|^{2}+2 J_{2}\left|A_{3}\right|^{2}\right) A_{1}+J_{3} A_{1}^{* 2} A_{3} \exp (i \delta \beta z)\right], \\
& \frac{\partial A_{3}}{\partial z}=i n^{(2)} k_{1}\left[\left(6 J_{2}\left|A_{1}\right|^{2}+3 J_{5}\left|A_{3}\right|^{2}\right) A_{3}+J_{3}^{*} A_{1}^{3} \exp (-i \delta \beta z)\right],
\end{aligned}
$$

where $\delta \beta=\beta_{3}-3 \beta_{1}$ is the phase mismatch, $k_{1}=\omega_{1} / c=2 \pi / \lambda_{1}$ is the propagation constant of the pump in vacuum, $n^{(2)}$ is the nonlinear refractive index coefficient, and $J_{i}$ are nonlinear overlap integrals (given in detail in Ref. [1]).

Fig. 1 shows the effective index curves for the fundamental pump mode $\operatorname{HE}_{11}\left(\omega_{1}\right)$ and fundamental third harmonic mode $\operatorname{HE}_{11}\left(3 \omega_{1}\right)$ in a silica microfibre for $\lambda_{1}=1.55 \mu \mathrm{m}$. At the microfibre diameter of $550 \mathrm{~nm}$ where $n_{1}^{\text {eff }}=1.012$ and 
$n_{3}^{\text {eff }}=1.334$, the phase mismatch is $\delta \beta=3.9 \times 10^{6} \mathrm{~m}^{-1}$. As this phase mismatch is large (comparable to $k_{1}$ ), the term $\exp (-i \delta \beta z)$ in Eq.(2b) oscillates very fast and prevents the fundamental mode harmonic from growing. Thus, efficient THG between the fundamental mode of the pump and the fundamental mode of the harmonic would be impossible without employing special methods to overcome such a large phase mismatch, as described next.

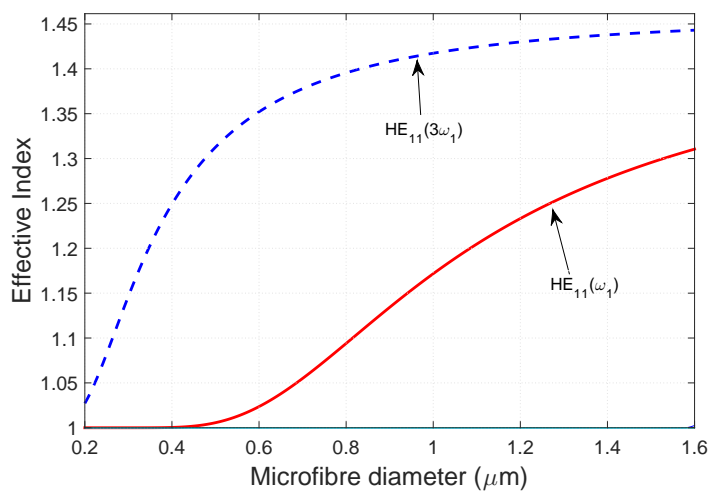

Fig. 1. Effective index curves for the fundamental pump mode $\operatorname{HE}_{11}\left(\omega_{1}\right)$ (solid red) and the fundamental third harmonic mode $\operatorname{HE}_{11}\left(3 \omega_{1}\right)$ (dashed blue) in a silica microfibre for $\lambda_{1}=1.55 \mu \mathrm{m}$.

If we introduce a weak counter-propagating light field $[4,5]$ at the fundamental frequency $\omega_{1}$ into the fibre, the superposition of the forward-travelling and weak counter-propagating beams can be described by a forward-propagating field that is modulated in both amplitude and phase along the direction of propagation with a periodicity of half the laser wavelength [4], that is,

$$
E_{1}(x, y, z, t)=A_{1 t}(z) F_{1}(x, y) \exp \left[i\left(\beta_{1 t} z-\omega_{1} t\right)\right]+\text { c.c. },
$$

where $\beta_{1 t}=\beta_{1}+\beta^{\prime}(z)$, and $\beta^{\prime}(z)$ has the form

$$
\beta^{\prime}=-\frac{2 r \beta_{1} \cos \left(2 \beta_{1} z\right)+2 r^{2} \beta_{1}}{1+2 r \cos \left(2 \beta_{1} z\right)+r^{2}} .
$$

Thus the actual total phase mismatch is $\delta \beta_{t}=\beta_{3}-3 \beta_{1 t}=\beta_{3}-3 \beta_{1}-3 \beta^{\prime}(z)$ and hence $\beta^{\prime}(z)$ acts as a compensative detuning which we can use to enhance the THG conversion. Importantly, the variation of $\beta^{\prime}(z)$ along the propagation distance can be adjusted by changing the ratio of the backward-forward field amplitudes $r=A_{1}^{-} / A_{1}^{+}$.

\section{Simulation results}

Figure 2 shows the compensative and total detuning against propagating distance when the backward-forward amplitudes ratio is $r=0.14$. The total detuning periodically approaches and even reaches zero, and at these positions, the harmonic power can be built up.

We calculate the third harmonic power in the presence of such a counter-propagating field by solving Eq.(2) numerically, and the results are given in Fig. 3. For small $r$, it is assumed that the counter-propagating pump generates a negligible counter-propagating harmonic signal. In the situation $r=0$, i.e. with only the forward pump, the harmonic power remains very low, but when the counter-propagating field is introduced (though only with $r=0.14$, or about $2 \%$ power of the forward pump) the harmonic power grows along the propagating direction, and in a microfibre tens of micrometres in length, the conversion efficiency obtains an enhancement of about one order of magnitude.

\section{Conclusion}

We have presented a scheme to enhance the fundamental-mode third harmonic generation efficiency in microfibres. By introducing an appropriate weak counter-propagating beam, the phase mismatch between the fundamental modes of the pump and third harmonic is partly compensated, enabling the harmonic power to grow along the propagation direction. This scheme offers an alternative idea for efficient harmonic generation by modifying the pump beam, and could potentially be applied to other small core waveguides such as wagon-wheel holey fibres or integrated waveguides. 


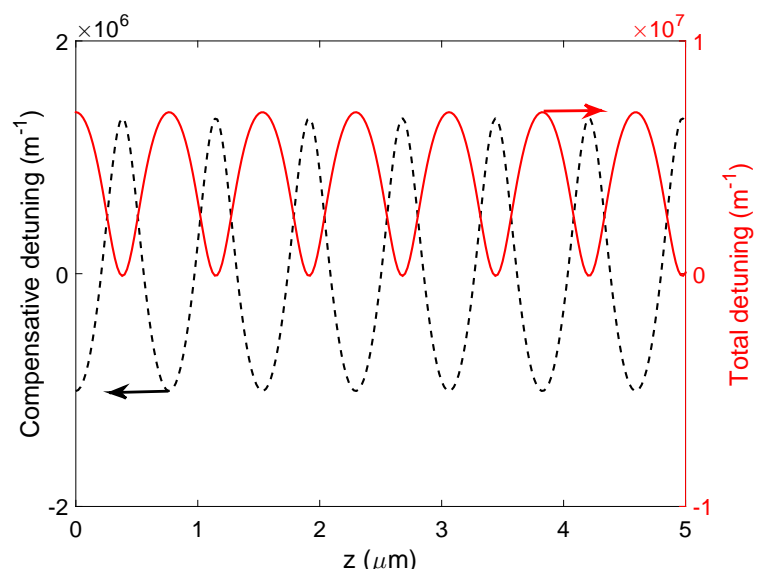

Fig. 2. The compensative detuning $\beta^{\prime}(z)$ introduced by the backward beam (black dashed curve) and the total detuning $\delta \beta_{t}$ (red solid curve) against propagating distance $z$, when the backward-forward amplitude ratio is $r=0.14$.

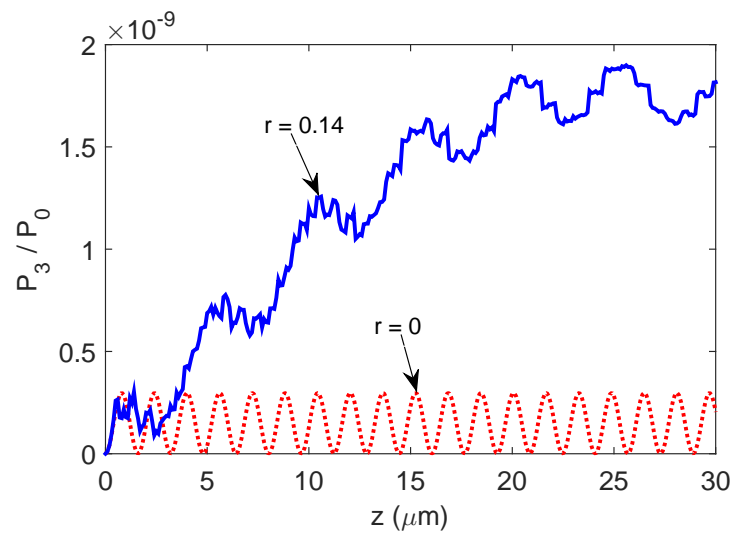

Fig. 3. The third harmonic generation power against the propagating distance $z$. The pump power $P_{0}=1 \mathrm{~kW}$, nonlinear overlap integral $J_{3}=0.31 \mu \mathrm{m}^{-2}, \lambda_{1}=1.55 \mu \mathrm{m}$, and $n^{(2)}=2.7 \times 10^{-20} \mathrm{~m}^{2} / \mathrm{W}$.

\section{Acknowledgments}

This work is supported by the National Natural Science Foundation of China (Grant No.11204043) and the Scholarship Fund of China (Grant No.201308440325).

\section{References}

1. V. Grubsky and A. Savchenko, "Glass micro-fibers for efficient third harmonic generation," Opt. Express 13, 6798-6806 (2005).

2. T. Lee, Y. Jung, C. A. Codemard, M. Ding, N. G. R. Broderick and G. Brambilla, "Broadband third harmonic generation in tapered silica fibres," Opt. Express 20, 8503-8511 (2012).

3. T. Wu, Y. Sun, X. Shao, P. Shum and T. Huang, "Efficient phase-matched third harmonic generation in an asymmetric plasmonic slot waveguide," Opt. Express 22, 18612-18624 (2014).

4. X. Zhang, A. L. Lytle, T. Popmintchev, X. Zhou, H. C. Kapteyn, M. M. Murnane and O. Cohen, "Quasiphase-matching and quantum-path control of high-harmonic generation using counterparopagating light," Nature Phys. 3, 270-275 (2007).

5. J. Peatross, S. Voronov and I. Prokopovich, "Selective zoning of high harmonic emission using counterpropagating light,” Opt. Express 1, 114-125 (1997). 